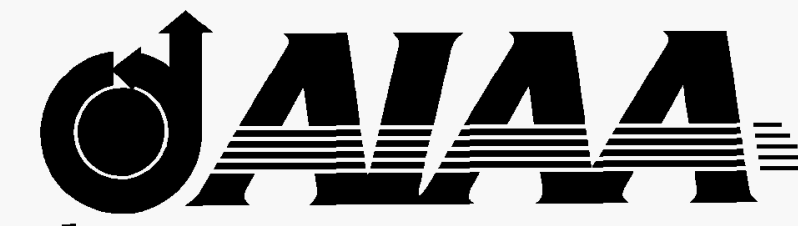

AIAA 98-2850

Mars Ascent Vehicle

Flight Analysis

P. N. Desai

R. D. Braun

W. C. Engelund

F. M. Cheatwood

NASA Langley Research Center Hampton, VA

J. A. Kangas

Jet Propulsion Laboratory,

California Institute of Technology

Pasadena, CA

7th AIAAIASME Joint Thermophysics and Heat Transfer Conference June 15-18, 1998 / Albuquerque, NM 


\title{
Mars Ascent Vehicle Flight Analysis
}

\author{
32nd AIAA Thermophysics Conference \\ June 15-18, 1998 \\ Albuquerque, NM.
}

\author{
P. N. Desai* \\ R. D. Braun* \\ W. C. Engelund* \\ F. M. Cheatwood* \\ NASA Langley Research Center \\ Hampton, VA 23681-2199
}

\author{
J. A. Kangas*** \\ Jet Propulsion Laboratory, California Institute of Technology \\ Pasadena, CA 91109
}

\begin{abstract}
The scientific objective of the Mars Surveyor Program 2005 mission is to return Mars rock, soil, and atmospheric samples to Earth for detailed analysis. The present investigation focuses on design of Mars Ascent Vehicle for this mission. Aerodynamic, aerothermodynamic, and trajectory design considerations are addressed to assess the ascent configuration, determine aerodynamic stability, characterize thermal protection system requirements, and ascertain the required system mass. Aerodynamic analysis reveals a subsonic static instability with the baseline configuration; however, stability augmentation options are proposed to mitigate this problem. The ascent aerothermodynamic environment is shown to be benign (on the order of the sea-level boiling point of water on Earth). As a result of these low thermal and pressure loads, a lightweight, low rigidity material can be employed as the aftbody aerodynamic shroud. The required nominal MAV lift-off mass is $426 \mathrm{~kg}$ for a December 2006 equatorial launch into a $300-\mathrm{km}$ circular orbit with 30-degree inclination. Off-nominal aerodynamic and atmospheric conditions are shown to increase this liftoff mass by approximately $10 \%$. Through performance of these analyses, the Mars Ascent Vehicle is deemed feasible with respect to the current mission mass and size constraints.

*Aerospace Engineer, Vehicle Analysis Branch, Space Systems and Concepts Division, Senior Member AIAA.

**Aerospace Engineer, Navigation \& Flight Mechanics Section, Systems Division.

Copyright (C) 1998 American Institute of Aeronautics and Astronautics, Inc. No copyright is asserted in the United States under Title 17, U.S. Code. The U.S. Government has a royaltyfree license to exercise all rights under the copyright claimed herein for Governmental purposes. All other rights are reserved by the copyright owner.
\end{abstract}

\section{NOMENCLATURE}

\begin{tabular}{|c|c|}
\hline$\alpha$ & Angle of attack \\
\hline APAS & Aerodynamic Preliminary Analysis System \\
\hline $\mathrm{C}_{\mathrm{D}}$ & drag coefficient \\
\hline CFD & computational fluid dynamics \\
\hline c.g. & center-of-gravity \\
\hline $\mathrm{C}_{\mathrm{m}_{0}}$ & static pitch stability coefficient \\
\hline GRAM & Global Reference Atmospheric Model \\
\hline HABP & Hypersonic Arbitrary Body Program \\
\hline Isp & specific impulse \\
\hline LAURA & $\begin{array}{l}\text { Langley Aerothermodynamic Upwind } \\
\text { Relaxation Algorithm }\end{array}$ \\
\hline $\mathrm{M}$ & Mach number \\
\hline MAV & Mars Ascent Vehicle \\
\hline MMH & Monomethyl Hydrazine \\
\hline MON-25 & $\begin{array}{l}\text { mixture of Nitrogen Textroxide and } \\
\text { Nitrogen Monoxide }\end{array}$ \\
\hline MSP & Mars Surveyor Program \\
\hline $\mathrm{N}$ & North \\
\hline OTIS & Optimal Trajectories by Implicit Simulation \\
\hline$p$ & surface pressure \\
\hline & free-stream pressure \\
\hline POST & Program to Optimize Simulated Trajectories \\
\hline q & heat rate \\
\hline Q-Alpha & $\begin{array}{l}\text { product of dynamic pressure and angle } \\
\text { of attack }\end{array}$ \\
\hline $\mathrm{RCS}$ & reaction control system \\
\hline S & South \\
\hline 3-D & three-dimensional \\
\hline $\mathrm{T}_{\mathrm{w}}$ & equilibrium wall temperature \\
\hline . & radial location along vehicle \\
\hline & long vehicle \\
\hline
\end{tabular}




\section{INTRODUCTION}

The primary scientific objective of the Mars Surveyor Program 2005 (MSP '05) mission is to acquire and return Mars rock, soil, and atmospheric samples to Earth for detailed investigation. ${ }^{1}$ This mission will crown a decade-long Mars exploration program and is the first in a sequence of planned sample-return missions. ${ }^{2}$ This flight project is likely the most challenging robotic exploration mission to date, requiring the development and integration of a Mars Lander, Mars rover, Mars Ascent Vehicle (MAV), Mars Orbiter, and Earth-Return ${ }^{3}$ Capsule under stringent cost and schedule constraints. The present paper describes a set of preliminary mission design analyses performed on the MAV. Aerodynamic, aerothermodynamic, and trajectory analyses are performed to assess the ascent configuration, obtain preliminary indications of aerodynamic stability, determine thermal protection system requirements, and ascertain the required ascent system mass. In conjunction with the flight system sizing performed at the Jet Propulsion Laboratory ${ }^{4}$ and NASA Marshall Space Flight Center, the MAV is deemed feasible within the MSP '05 mass and size constraints. This paper represents a status of work in progress; further refinement and trades are ongoing.

In the present mission architecture, the MAV, housed within the Mars Lander, is launched to Mars in September 2005. The Lander performs a direct entry, descent, and landing, touching down on the Mars surface in October 2006. Once on the Mars surface, the Rover is deployed to acquire and place the selected science samples in a canister on the MAV. After sealing the sample canister, the MAV transports this payload to low-Mars orbit where an autonomous rendezvous with the Mars Orbiter/Earth-Return Capsule is performed. (The Mars Orbiter and Earth-Return Capsule are either launched a month prior to the Mars Lander/MAV or on the same launch vehicle). The sample canister is transferred to the Earth-Return Capsule while in Mars orbit. Then, the Earth-return capsule departs Mars for Earth in July 2007, concluding the 9-month Mars operations sequence. Earth-return and recovery of the Mars samples occurs in May 2008.

A two-stage-to-orbit ascent system which relies on pressure-fed rocket propulsion using storable propellants is currently baselined (Fig. 1). This system uses Monomethyl Hydrazine (MMH) as fuel and a mixture of Nitrogen Textroxide and Nitrogen Monoxide (MON25 ) as the oxidizer. The first-stage vacuum specific impulse (Isp) is $323 \mathrm{sec}$, while the second-stage vacuum Isp is $308 \mathrm{sec}^{4}$ An optimal thrust profile is determined as part of the present investigation. First-stage propul-

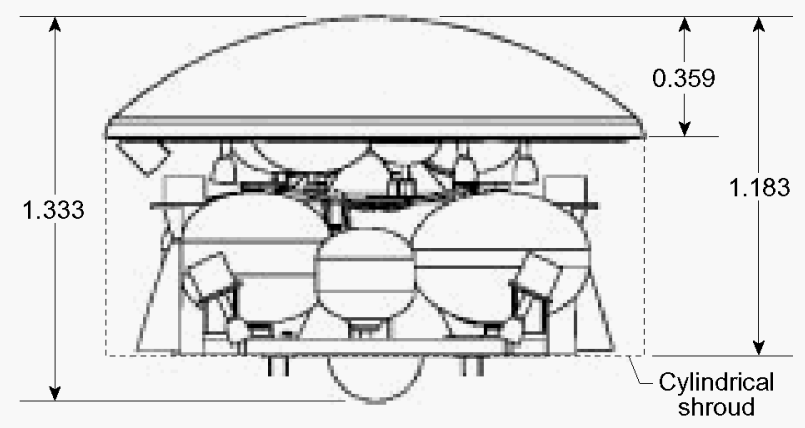

a. Lift-off configuration.

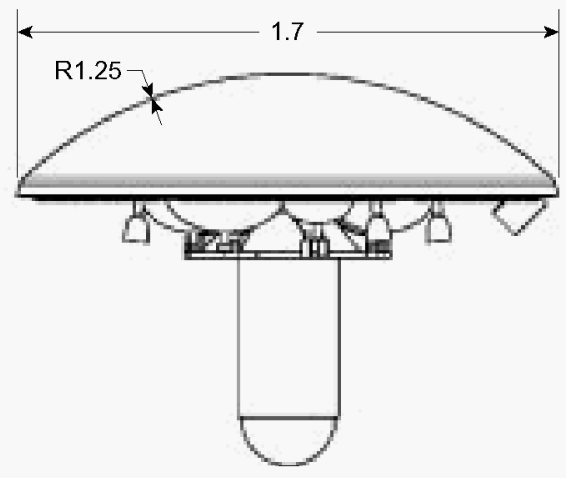

b. Second stage configuration.

Fig 1 Reference MAV configuration (dimensions in meters).

sion consists of two main engines complemented by four reaction-control system (RCS) thrusters to maintain the appropriate attitude. Second-stage propulsion is accomplished through the use of four additional RCS thrusters which provide axial thrust, as well as attitude control.

The basic reference aerodynamic shape for the Mars ascent vehicle is dictated primarily by packaging and volumetric constraints imposed by the Mars Lander (Ref. 4). The MAV is currently designed as a two stage launch system. However, unlike a typical "stacked" multistage system, the second stage of the MAV is placed within the first stage, in order to remain within the volumetric envelope constraint of the Lander. The complete configuration (shown in Fig. 1a) is a short, spherically blunted cylinder, approximately $1.2 \mathrm{~m}$ in length, with a maximum diameter of $1.7 \mathrm{~m}$, and a spherical nose with a $1.25 \mathrm{~m}$ constant radius. A cylindrical afterbody shroud is positioned around the first stage surrounding the payload, propellant and oxidizer tankage, ascent rocket motor, and associated hardware. The second stage (shown in Fig. 1b) is comprised of only the spherical aeroshell fairing, which protects the remaining tankage, payload, and hardware from the stagnation pressures and temperatures during 
the high Mach number portion of the ascent. The configuration continues to evolve. In this analysis, a $30 \mathrm{~kg}$ payload, consisting of $0.3 \mathrm{~kg}$ of science samples, a 2.7 $\mathrm{kg}$ sample canister, and an avionics module is assumed. ${ }^{5}$ Ascent originates at an equatorial site and terminates in a $300-\mathrm{km}$ circular orbit with a $30^{\circ}$ inclination. To satisfy MSP '05 mission constraints, the MAV must have a total mass less than approximately $500 \mathrm{~kg}$.

\section{ANALYSIS}

\section{Aerodynamics}

The aerodynamic characteristics of the reference MAV configuration was estimated utilizing the Aerodynamic Preliminary Analysis System (APAS) ${ }^{6,7}$ code. APAS is a preliminary evaluation tool used to obtain rapid estimations of configuration aerodynamics, including static, dynamic, and control effectiveness for the longitudinal and lateral-directional characteristics of arbitrary threedimensional configurations throughout the flight regimes. It is not a substitute for wind tunnel or more rigorous computational results, but rather provides an efficient means of estimating vehicle aerodynamic characteristics in the conceptual and preliminary design stages. In the subsonic and low supersonic regimes, APAS utilizes a combination of linear potential methods, with empirical viscous and wave drag estimating techniques. In the supersonic through hypersonic flight regimes, a variety of classical theoretical and empirical impact pressure methods are available (e.g. Newtonian methods, tangent cone, tangent wedge, etc.), along with various approximate boundary layer relations. The supersonic/hypersonic analysis module used within APAS is a modified version of the Hypersonic Arbitrary Body Program (HABP) Mark III. In this study, all of the APAS solutions at Mach numbers (M) below 2.5 were computed using the linear potential code methods. At Mach numbers above 2.5, the hypersonic impact methods were utilized.

Since the reference configuration has a low fineness ratio, its blunt body geometry deviates rather substantially from the assumptions associated with linear perturbation analysis methods employed within APAS. In an effort to improve the present aerodynamic predictions, a set of correction increments were developed based on analyzing a similar flight proven configuration. The Discoverer shape (Fig. 2) was analyzed using the same APAS methods, and the results were compared with the existing Discoverer aerodynamic database. The increments obtained from this comparison were then applied to the APAS estimates of the reference vehicle to enhance the aerodynamic characteristics for Mach numbers below 2.5 and the angle-of-attack range of interest.

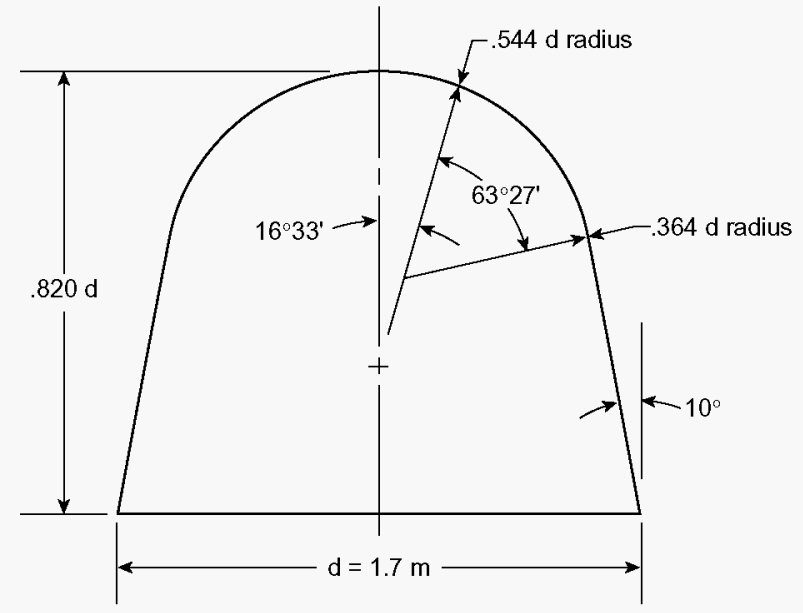

Fig. 2 Discoverer configuration.

The Discoverer shape was studied in the early 1960's for use as a possible planetary entry instrument probe to study planetary atmospheres; it was also utilized as an Earth entry capsule as part of the National Reconnaissance Office's Corona program. ${ }^{8-11}$ The configuration (shown in Fig. 2) is a short spherically blunted cone with a $10^{\circ}$ semivertex angle aftbody. The configuration shape was scaled such that the maximum base diameter was equivalent to that of the reference configuration $(1.7 \mathrm{~m})$. This shape, because of its less blunt nose and slightly flared aftbody, has significantly less aerodynamic drag and greater inherent static stability than that of the reference MAV configuration. In fact, it is statically stable for all Mach numbers at center-of-gravity (c.g.) locations up to $59 \%$ of the reference length. In addition, it has been extensively tested, and has made in excess of 100 successful Earth atmospheric entries as part of the Corona program. As such, it has an established well characterized aerodynamic database. This shape does not however, meet the current Lander packaging and volumetric constraints for the MAV system.

\section{Aerothermodynamics}

The aerothermodynamics analysis was performed using the Langley Aerothermodynamic Upwind Relaxation Algorithm (LAURA) ${ }^{12-14}$ which solves the thinlayer Navier-Stokes equations. LAURA is a state-of-the-art computational fluid dynamics (CFD) code which discretizes the governing equations within a domain defined by a structured volume grid. In the past, LAURA has been used at Mars for the Pathfinder ${ }^{15}$ and Microprobe ${ }^{16}$ missions and at Earth for numerous launch vehicle configurations. A volume grid consisting of 42 cells in the axial direction, 18 cells in the circumferential direction, and 64 cells in the body-normal direction is 
employed about the vehicle geometry for this analysis. An eight-species, two-temperature gas model for Mars is employed, along with a radiative equilibrium wall temperature boundary condition. A super-catalytic material is specified for the vehicle, which forces recombination of the gas to free stream composition at the surface.

\section{Trajectory Simulation}

The trajectory analysis was performed using two three degree-of-freedom trajectory programs, the Program to Optimize Simulated Trajectories (POST) ${ }^{17}$ and the Optimal Trajectories by Implicit Simulation (OTIS) ${ }^{18}$ program. POST solves the trajectory optimization problem by numerically integrating the equations of motion, while OTIS utilizes a collocation scheme to discretize the equations of motion. Both codes are used to model vehicle flight from Mars surface to orbit with the objective of minimizing the vehicle lift-off mass subject to inflight constraints. The ascent trajectory simulation includes Mars atmospheric (Mars-GRAM) ${ }^{19}$ and gravitation models, as well as vehicle aerodynamic, mass property, and propulsion models.

\section{RESULTS \& DISCUSSION}

\section{Aerodynamics/Configuration Trades}

Aerodynamic Characteristics - Drag coefficient data (at zero angle-of-attack) as a function of Mach number for both the reference shape and the Discoverer configuration are shown in Fig. 3. The reference configuration has roughly twice the aerodynamic drag as the Discoverer shape at all Mach numbers. However, since the dynamic pressure is relatively low as a result of the thin Martian atmosphere, aerodynamic drag is not a large

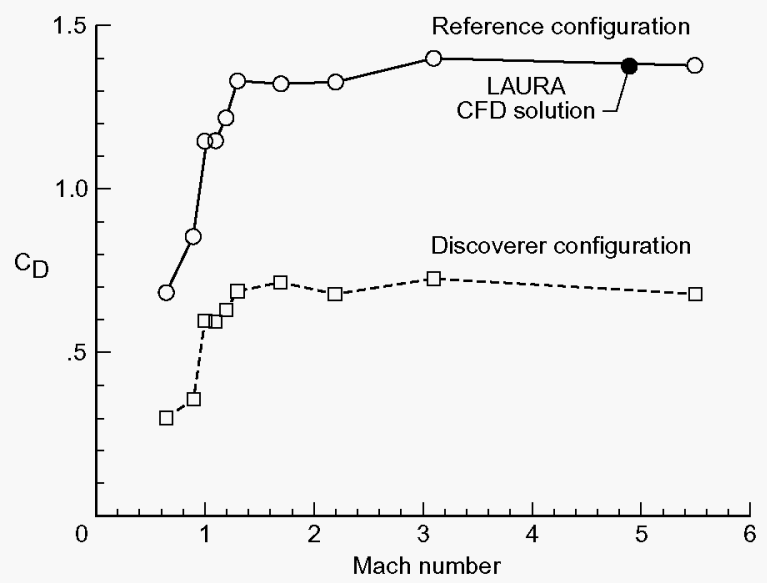

Fig. 3 Drag coefficient comparison between the reference $M A V$ and Discoverer configurations.

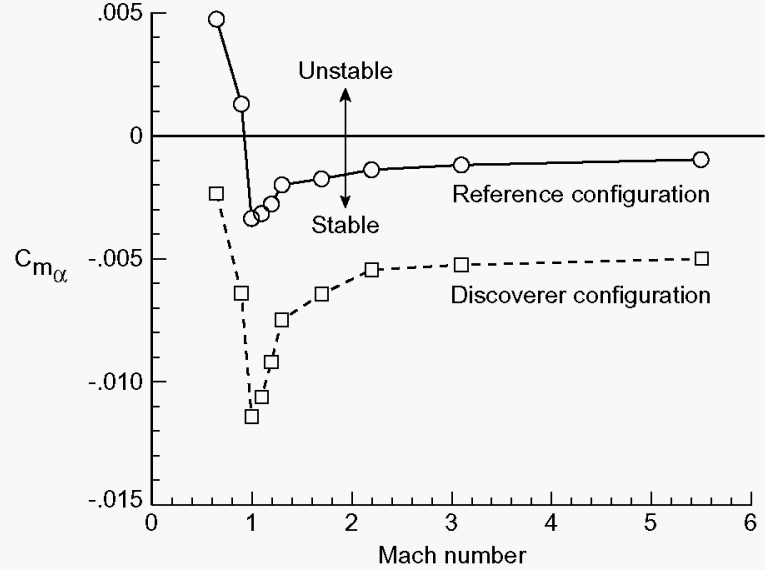

Fig. 4 Pitch stability comparison between the reference $M A V$ and Discoverer configurations.

driver in the overall MAV design optimization process. The predicted drag coefficient value from the result of a Navier-Stokes CFD solution at the reference configuration Mach 5 condition (discussed later in this paper) is also shown. This result agrees within approximately $2 \%$ of the APAS prediction.

Using a preliminary mass properties model, estimates of the vehicle c.g. are developed over the reference flight trajectory, and an assessment of the static pitch stability $\left(\mathrm{C}_{\mathrm{m}_{\alpha}}\right)$ about the vehicle c.g. as a function of ascent Mach number is made (Fig. 4). As seen, the reference shape is statically unstable (positive values of $\mathrm{C}_{\mathrm{m}_{\alpha}}$ ) during the subsonic portion of the ascent. As it passes through the transonic regime, it becomes statically stable and remains so, at low levels, through the supersonic portion of the ascent. The Discoverer shape, shown for comparison, is statically stable throughout all phases of flight for the same relative c.g. position history. Again, due to the low dynamic pressures associated with the ascent through the Martian atmosphere, the requirement to have a statically stable vehicle may not exist. The impact of this MAV instability upon the ascent is being assessed in a six degree-of-freedom stability analysis. This assessment, which is beyond the scope of the current paper, is being conducted in order to determine the adequacy of the current reaction control system, as well as the associated propellant requirements.

If stability has a pronounced impact on the MAV RCS propellant requirements, the reference configuration would require some form of stability augmentation. One option to provide pitch and yaw stability is the addition of small deployable aerodynamic fins. To remain within the volumetric and packaging constraints of the system, the fins could be stored in a folded arrangement as indi- 

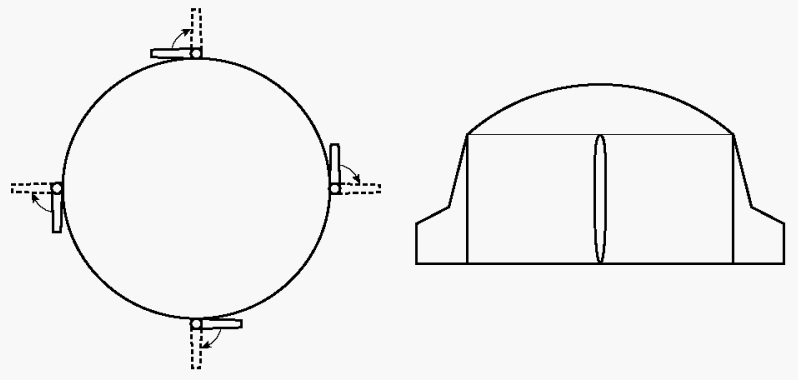

\section{a. Folded arrangement. $\quad$ b. Deployed arrangement.}

Fig. 5 Fin option for enhancing stability of reference $M A V$ configuration.

cated in Fig. 5a. Prior to the ascent, the fins would be deployed in a standard cruciform arrangement providing a stabilizing pitch and yaw moment over the trajectory until first stage separation. Reference configuration aerodynamics were estimated with the set of fins as shown in Fig. 5b, and the resulting stabilizing effect is indicated as a function of Mach number by the $\mathrm{C}_{\mathrm{m}_{\alpha}}$ comparison shown in Fig. 6. The addition of the fin set decreases the value of $\mathrm{C}_{\mathrm{m}_{\alpha}}$ (thereby increasing the pitch stability) over the entire Mach range. With fins, the reference MAV configuration exhibits a similar level of stability as the Discoverer shape. Trade studies are being performed to assess the added complexity of fin aerodynamic surfaces vs. utilization of reaction control vs. external shape modifications. A combination of these three stability augmentation methods is likely.

Uncertainties - Based on the preliminary nature of the calculations, uncertainties on the aerodynamic characteristics (lift, drag, and pitching moment coefficient data) were estimated to be $\pm 30 \%$ below Mach 2 and $\pm 10 \%$

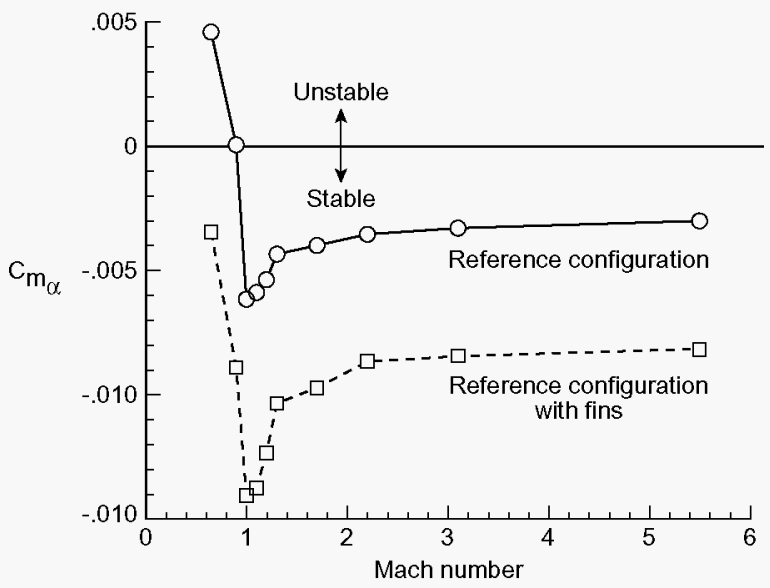

Fig. 6 Pitch stability comparison with and without fins for the reference configuration. above Mach 2. These uncertainties are used to quantify the sensitivity of the MAV system design to aerodynamic performance in the next section. These uncertainty values can be reduced by improving the fidelity of the aerodynamic model through wind tunnel tests and additional CFD analyses.

\section{Performance Optimization}

An optimal ascent from the surface of Mars to the rendezvous orbit was determined to assess the propulsion requirements of the MAV. Engine parameters (e.g., first stage main engine thrust magnitude, area ratio, and Isp, and second stage RCS thruster magnitude) and the ascent pitch attitude history of the MAV are optimized to determine the minimal lift-off mass required to ascend to the desired orbit. Since, a Mars lift-off date or landing site for the MSP '05 mission has not been selected, an equatorial launch occurring in the middle of December 2006 is baselined in this analysis. A launch site from a latitude between $30^{\circ} \mathrm{N}$ and $15^{\circ} \mathrm{S}$ is likely due to the scientific interest in the Mars ancient highland regions. An atmosphere was generated from MarsGRAM for this launch period. The MAV is injected into a transfer orbit having an apoapsis of $300 \mathrm{~km}$ and an inclination of $30^{\circ}$. Upon arrival at apoapsis, a circularization maneuver is performed to initiate rendezvous and subsequent transfer of the sample canister to the awaiting Earth Return Capsule.

The stage masses of the MAV are determined through a set of mass estimation relationships obtained from the Jet Propulsion Laboratory. ${ }^{4}$ These mass estimation equations relate the engine parameters and ascent propellant to the overall dry mass of each stage. The permitted range of variation of the engine parameters is:

\section{Stage 1}

Engine Vacuum Thrust: 400-800 lbf (per engine) Area Ratio: 50-150 (per engine)

Stage 2

Thruster Vacuum Thrust: 25-50 lbf (per thruster)

Note, the stage 1 engine area ratio alters the stage 1 engine nominal Isp by $-2 \%$ to $+1 \%$ for the 50 to 150 area ratio range.

Using the mass estimation relationships for the MAV dry stages and the range of engine parameters listed above, optimal ascent trajectories were calculated using both the POST and OTIS trajectory codes. For an inflight constraint on Q-Alpha of $\pm 335 \mathrm{~N}-\mathrm{deg} / \mathrm{m}^{2}$, the liftoff mass of the MAV is found to be approximately 426 $\mathrm{kg}$. Q-Alpha, which is the product of dynamic pressure 
and angle of attack, was constrained to limit structural loads during the ascent. Table 1 summarizes the optimal engine parameters as well as the MAV stage mass breakdown obtained from the POST and OTIS simulations. The lift-off mass determined by these two independent simulations is within $0.3 \%$ of each other, and the engine parameters optimized to identical values. Note, the optimal stage 1 engine thrust and area ratio were found to be at their lower and upper bounds, respectively.

Figures 7-10 show the ascent trajectory obtained by the two simulations. As seen, good agreement exists between the two trajectory programs. Figures 7 and 8 show the altitude and velocity time histories, respectively, of the ascent to the initiation of the transfer orbit. First stage separation occurs at approximately $180 \mathrm{sec}-$ onds (Mach 6.6) at a dynamic pressure of $34 \mathrm{~N} / \mathrm{m}^{2}(0.7$ $1 \mathrm{bf} / \mathrm{ft}^{2}$ ). Figures 9 and 10 show the angle of attack and corresponding Q-Alpha profiles, respectively. Note, a better comparison of the angle of attack and Q-Alpha

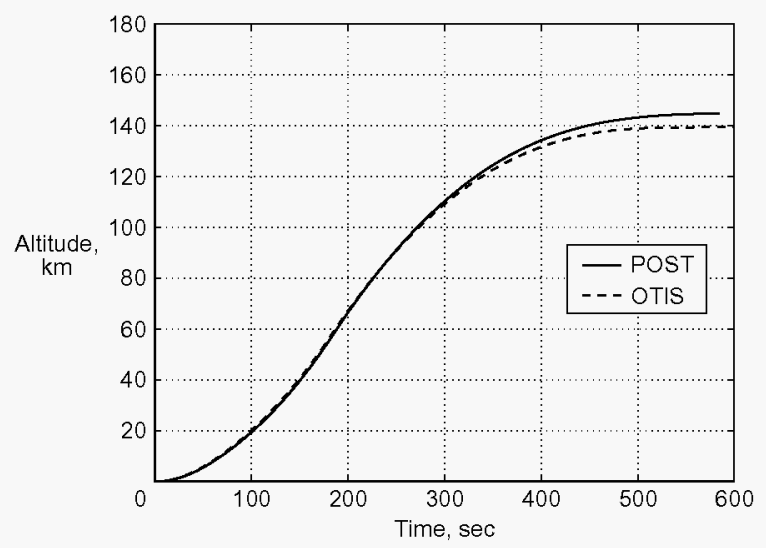

Fig. 7 Altitude comparison between POST and OTIS.

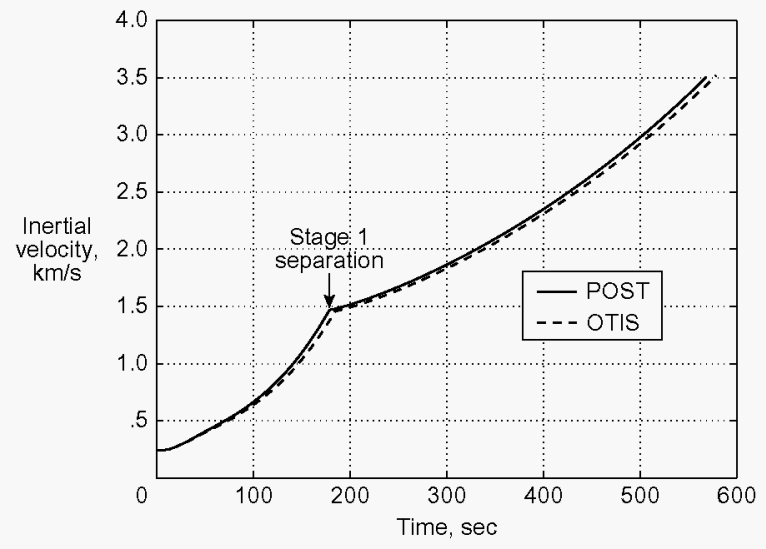

Fig. 8 Inertial velocity comparison between POST and OTIS.
Table 1. POST and OTIS Trajectory Results

\begin{tabular}{lcc}
\hline \hline Engine Parameters & POST & OTIS \\
Stage 1 engine vacuum thrust, lbf & $400($ each) & 400 \\
Stage 1 engine area ratio & 150 & 150 \\
Stage 1 engine Isp, sec & 327 & 327 \\
State 2 thruster vacuum thrust, lbf & 30 (each) & 30 \\
\hline Stage Mass Breakdown & & \\
Stage 1 dry mass, kg & 88.1 & 89.0 \\
Stage 1 propellant mass, kg & 207.4 & 205.3 \\
Stage 2 dry mass, kg & 31.6 & 32.1 \\
Stage 2 propellant mass, kg & 68.8 & 69.4 \\
Payload mass & 30.0 & 30.0 \\
Total MAV lift-off mass & 425.9 & 427.0 \\
\hline \hline
\end{tabular}

time histories can be achieved, if additional discretization points are added to the OTIS simulation. However, in light of the excellent lift-off mass agreement, further refinement of the OTIS trajectory was not warranted. The

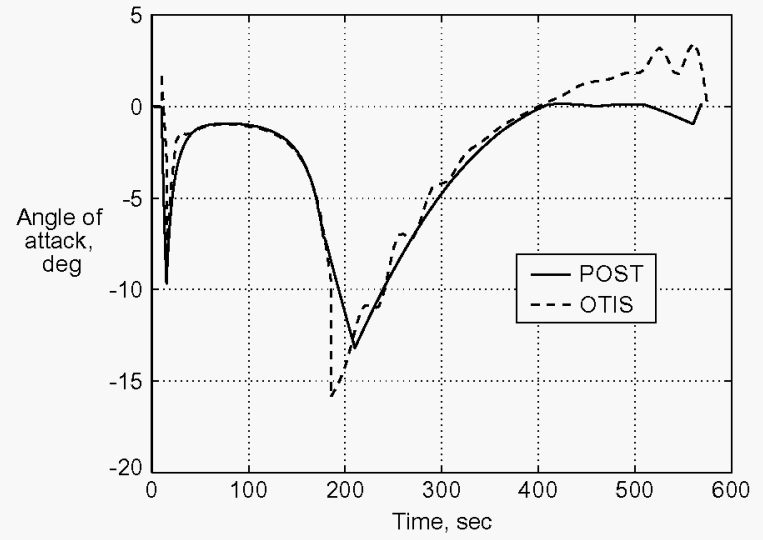

Fig. 9 Angle-of-attack comparison between POST and OTIS.

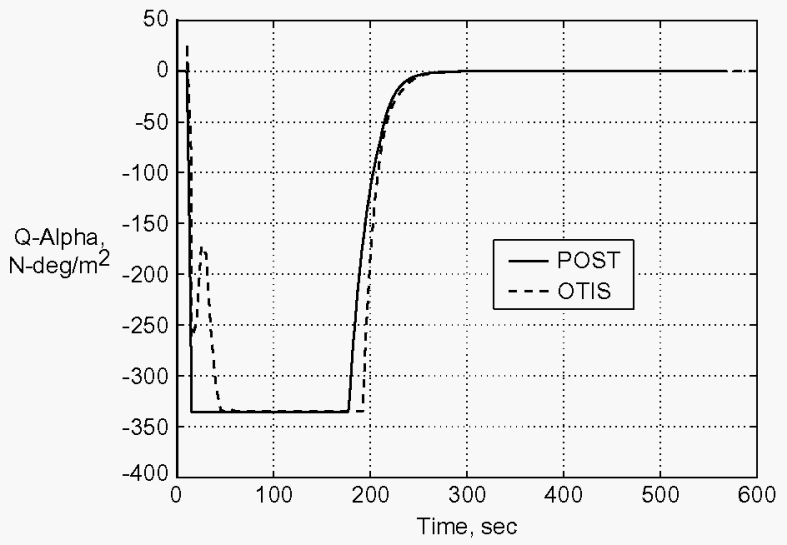

Fig. 10 Q-alpha comparison between POST and OTIS. 
angle of attack obtained is as high as $-15^{\circ}$ late in the ascent. This high attitude is not of great concern, since the dynamic pressure is low $\left(<30 \mathrm{~N} / \mathrm{m}^{2}\right)$ at this flight condition. The peak acceleration and dynamic pressure during the ascent are 1.7 Earth-g's and $361 \mathrm{~N} / \mathrm{m}^{2}\left(7.5 \mathrm{lbf} / \mathrm{ft}^{2}\right)$, respectively. The optimal transfer orbit is $144 \mathrm{~km}$ by 300 $\mathrm{km}$, producing a coast time to apoapsis of about $53 \mathrm{~min}-$ utes. The total ascent time from lift-off to the rendezvous orbit is approximately 63 minutes. A direct ascent to a $300 \mathrm{~km}$ circular orbit is also being analyzed.

\section{Aerothermodynamics}

A three-dimensional (3-D) computational aerothermodynamic solution was calculated at the predicted peak heating flight conditions of Mach 4.8 and angle of at$\operatorname{tack}(\alpha)$ of $5^{\circ}$. Figure 11 shows flooded color contours of the surface heating, and corresponding radiative equilibrium wall temperature, for the 3-D case.

Figures 12 and 13 show the symmetry plane heating distributions as functions of $\mathrm{z}$ and $\mathrm{x}$, respectively. A stagnation value of less than $0.1 \mathrm{~W} / \mathrm{cm}^{2}$ is predicted. The windside shoulder heating is seen to be approximately 85 percent of the stagnation value, while the leeside is at 70 percent. Thus, for angles-of-attack on the order of $5^{\circ}$ at peak heating, a monolithic heat shield sized to the stagnation conditions should be adequate for the entire forebody of the vehicle. Figures 14 and 15 show the corresponding radiative equilibrium wall temperature distributions as functions of $\mathrm{z}$ and $\mathrm{x}$, respectively, using a surface emissivity of 0.9 . The highest wall temperature observed is approximately $370 \mathrm{~K}$ at the stagnation point. Hence, the predicted maximum temperature is on the order of the boiling point of water at sea level on Earth.

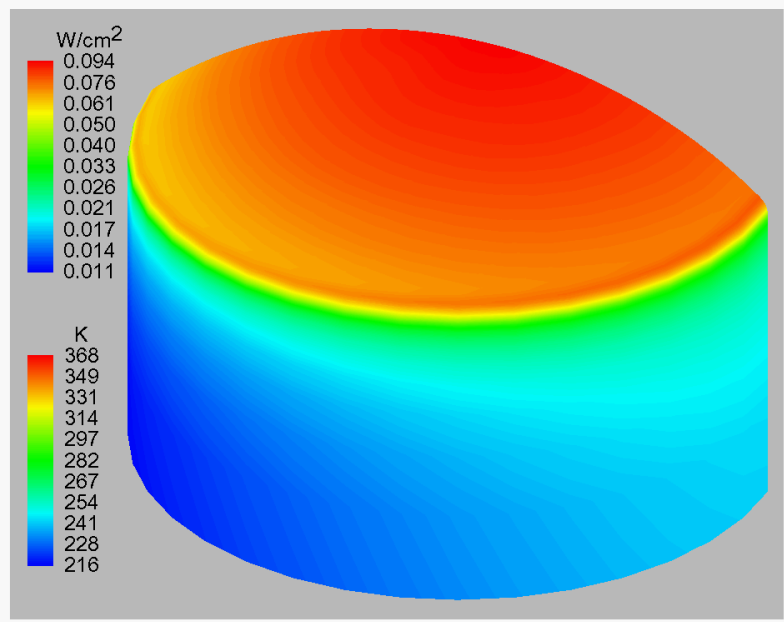

Fig. 11 Surface heating and equilibrium wall temperature contours at peak heating $(\alpha=5 \%$.

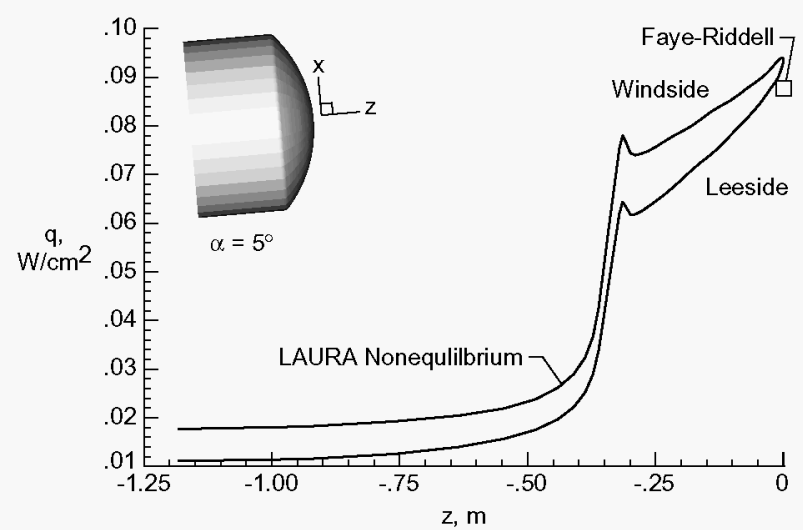

Fig. 12 Axial distribution of symmetry plane surface heating at peak heating.

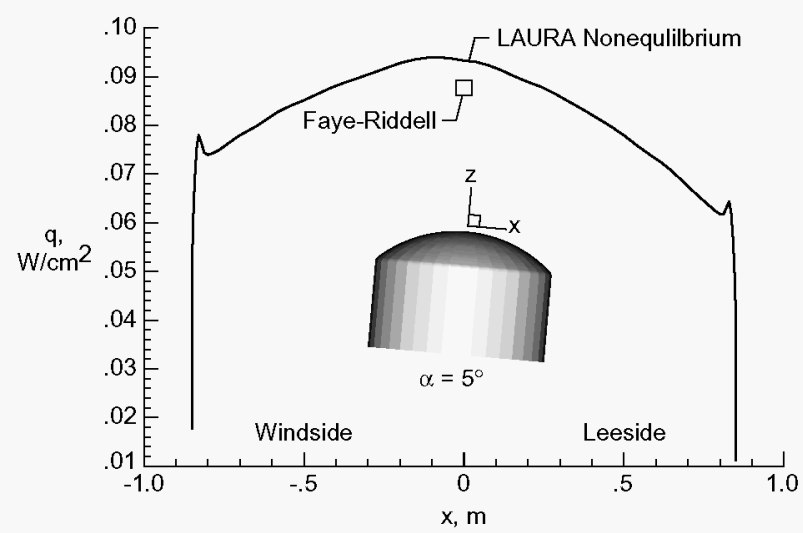

Fig. 13 Radial distribution of symmetry plane surface heating at peak heating.

Also shown in Figs. 12 and 13 is a perfect gas FayeRiddell value obtained from the trajectory simulation; the corresponding radiative equilibrium wall temperature is shown in Figs. 14 and 15. The relatively good agreement between the CFD and empirical values lends to the definition of a ratio of the CFD surface distribution to the Faye-Riddell value. As the nominal trajectory evolves, this distribution of ratios can be used in conjunction with varying stagnation heating values obtained by FayeRiddell (from the trajectory simulation) to perform preliminary TPS sizing along the vehicle surface.

Figure 16 shows the surface pressure distribution (normalized by freestream value) over the MAV configuration. As seen in the figure, the pressure load for the MAV cylindrical shroud is near free-stream levels. As stated earlier, the maximum dynamic pressure experienced during the ascent is approximately $361 \mathrm{~N} / \mathrm{m}^{2}(7.5$ $\mathrm{lbf} / \mathrm{ft}^{2}$ ). Therefore, a light weight, low rigidity material can be employed for the cylindrical afterbody. 


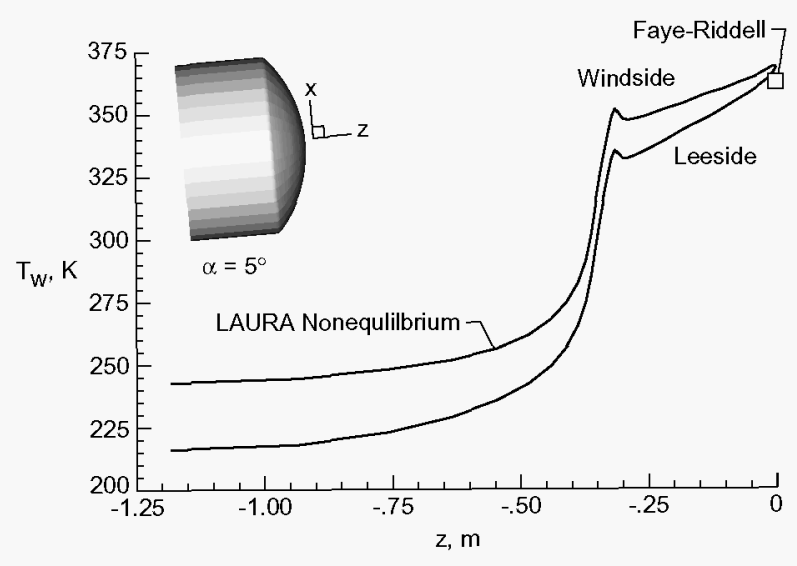

Fig. 14 Axial distribution of symmetry plane wall temperature at peak heating.

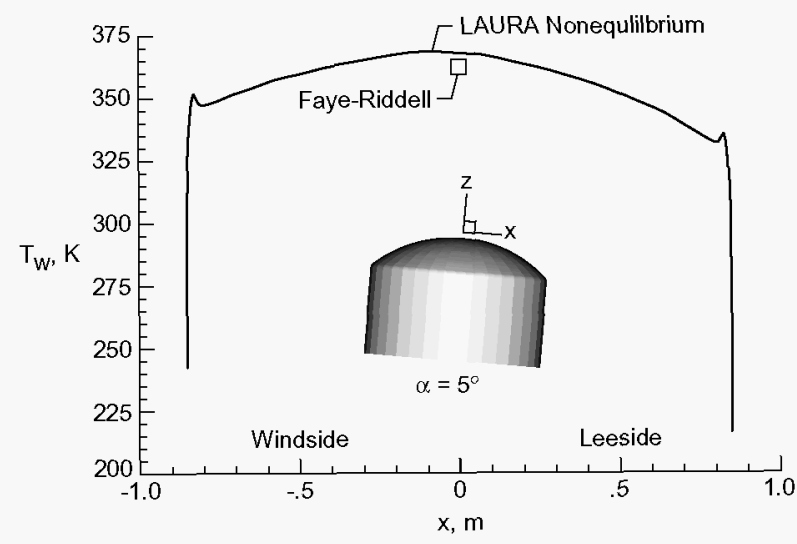

Fig. 15 Radial distribution of symmetry plane wall temperature at peak heating.

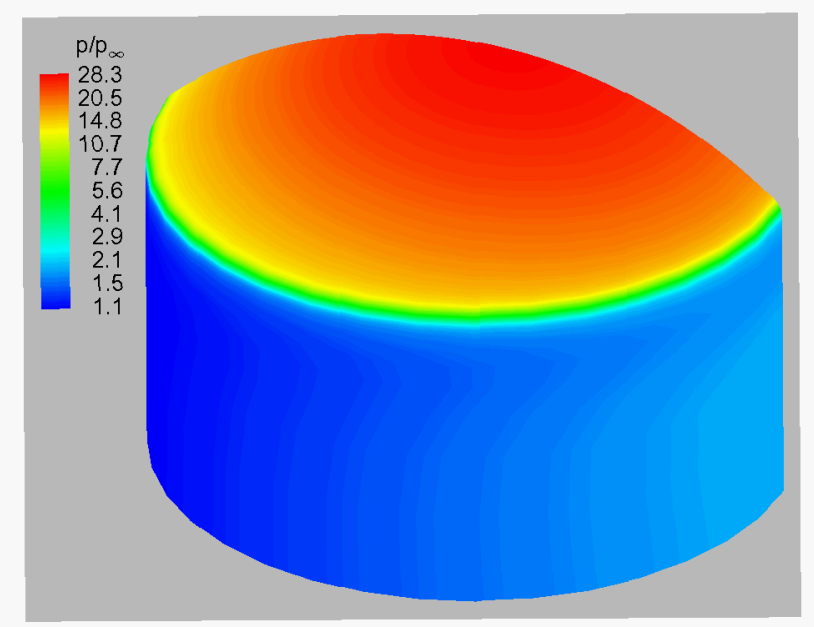

Fig. 16 Normalized surface pressure contours at peak heating $(\alpha=5 \%$.

\section{Dispersion Analysis}

The probability that nominal reference conditions will exist during the ascent is remote. The uncertainties in the MAV configuration and the ascent will have an impact on the MAV lift-off mass. Uncertainties in MAV performance (engine thrust and Isp), MAV mass properties (moments of inertia and c.g. location), MAV aerodynamics, guidance system, and Mars atmosphere must be accounted for. To determine the sensitivity of the current MAV design to off-nominal conditions, MAV aerodynamics and the Mars atmosphere are dispersed to ascertain the impact on the lift-off mass.

The current MSP '05 mission profile calls for ascent of the MAV to take place between October 2006 and May 2007, depending upon the required rover sample acquisition time. Since this launch period encompasses different seasons, the variation in the Mars atmosphere can be large. Figure 17 illustrates this variation by comparing the atmospheric density between a mid-December 2006 (least dense) to mid-May 2007 (most dense) launch. The mean density for May 2007 is as much as $50 \%$ greater than the mean December 2006 density. If the launch occurs in May 2007, the nominal MAV mass would increase to $452.8 \mathrm{~kg}$; an increase of $27 \mathrm{~kg}(6.3 \%)$ over a December launch. Also shown in Fig. 16, is the $+3-\sigma$ variation in the mean density for mid-December '06 and mid-May' 07 which occur due to daily fluctuations and atmospheric prediction uncertainty. The mass increment resulting from these $+3-\sigma$ density values is calculated to be approximately $6 \mathrm{~kg}$ for both launch periods. Figure 18 plots the mass increases in the MAV (from the December 2006 launch value) due to variations in the Mars atmospheric density.

Also shown in Fig. 18, is the increase in mass (for the two launch periods) arising from an uncertainty in

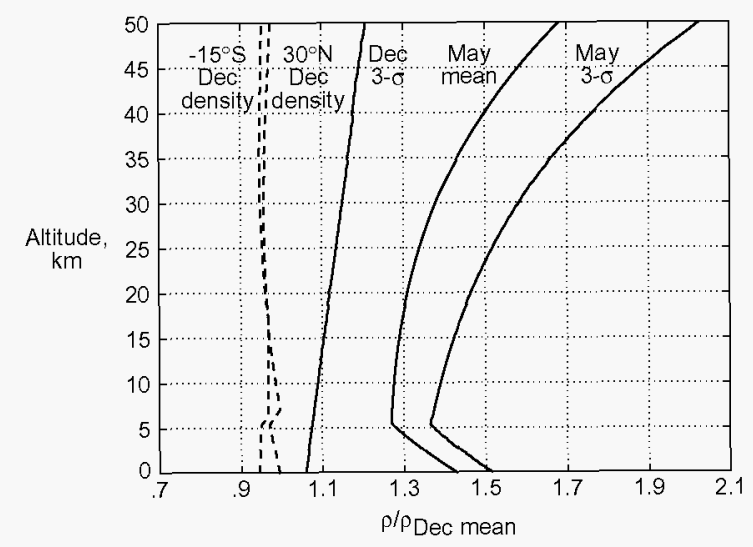

Fig. 17 Mars-GRAM density variation. 


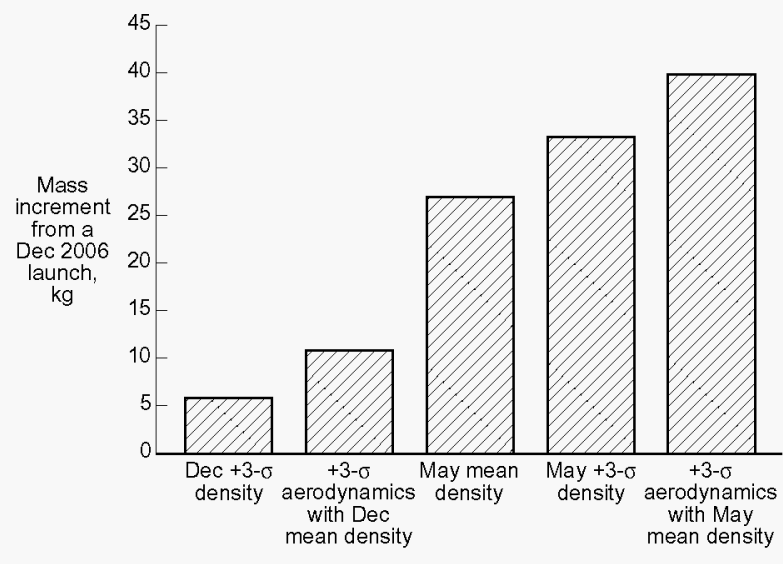

Fig. 18 Mass impact of applying atmospheric and aerodynamic uncertainties independently.

the aerodynamics of the MAV configuration. As mentioned previously, this uncertainty is estimated to be $\pm 30 \%$ for $M<2$ and $\pm 10 \%$ for $M>2$. As seen in Fig. 18, the impact of this uncertainty is approximately $11 \mathrm{~kg}$ and $13 \mathrm{~kg}$ for December and May launches, respectively. Taking the $\mathrm{L}$ norm of these mass increments (the $+3-\sigma$ density and the aerodynamics uncertainties) and adding it to the May 2007 launch mass provides an estimate on the overall MAV lift-off mass required, including margin. The resulting mass of the MAV is $467.2 \mathrm{~kg}$, an increase of approximately 10\% from the December 2006 launch value. The inclusion of additional uncertainties (e.g., stability, guidance, and mass properties) will increase these values, but it is likely that the MAV lift-off mass will remain below $500 \mathrm{~kg}$.

The mass impact of non-equatorial launches (between $30^{\circ} \mathrm{N}$ and $-15^{\circ} \mathrm{S}$ ) is determined to be negligible. In fact, launch from a $30^{\circ} \mathrm{N}$ latitude results in a lower MAV lift-off mass (by $3 \mathrm{~kg}$ ) than a December 2006 equatorial launch; the lift-off mass from a $-15^{\circ} \mathrm{S}$ latitude launch is found to be the same as that of a December 2006 equatorial launch. This outcome is a consequence of a lower density profile (by $5 \%$ ) at these latitudes as compared to the mean December 2006 equatorial density (as seen in Figure 17). For a $30^{\circ} \mathrm{N}$ latitude launch, the decrease in velocity (attained from the planet's rotation) due to the higher latitude is off-set by a lower drag loss during the ascent producing a lower overall lift-off mass. A similar result is obtained for a $-15^{\circ} \mathrm{S}$ latitude launch because a due East launch is not performed. Using the mean December 2006 equatorial density for these two off-equatorial launches results in a higher lift-off mass (as one would expect).

\section{SUMMARY}

This study characterizes preliminary aspects of the aerodynamics, aerothermodynamics, and trajectory performance of a proposed Mars Sample Return ascent vehicle. The analyses show that the current MAV design is within the present mass and geometric constraints imposed for the mission. The aerodynamic analysis of the MAV reveals a subsonic static instability. However, due to the thin Mars atmosphere and low dynamic pressures associated with the ascent, the requirement to have a statically stable vehicle is presently unclear. A six degree-of-freedom flight dynamics analysis is being performed to assess the impact of this instability on the overall MAV design. A stability augmentation option has been proposed to mitigate this potential problem.

The aerothermodynamic environment during ascent is found to be benign. The predicted maximum equilibrium wall temperature is on the order of the boiling point of water at sea level on Earth. The required nominal MAV lift-off mass is calculated to be $426 \mathrm{~kg}$ for a December 2006 launch. Off-nominal conditions during the ascent arising from uncertainties in vehicle aerodynamics and atmospheric properties are found to produce mass increases on the order of $10 \%$. Hence, the reference MAV design meets the present MSP '05 mission mass and size constraints with a mass margin of approximately $7 \%$. A Monte Carlo analysis is underway to assess the impact of additional uncertainties on the MAV lift-off mass.

\section{ACKNOWLEDGMENTS}

The authors would like to extend their appreciation to Carl Guernsey of the Jet Propulsion Laboratory for supplying the MAV mass estimation equations, and Larry Lee of the Jet Propulsion Laboratory and Greg Griffin of Swales Aerospace for providing the MAV geometry and mass property information.

\section{REFERENCES}

1) Matousek, S., Adler, M., and Lee, W., "A Few Good Rocks: The Mars Sample Return Mission Architecture," AIAA Paper 98-4282, Aug. 1998.

2) Shirley, D.L., and McCleese, D.J., "Mars Exploration Program Strategy: 1995-2020," AIAA 96-0333, Jan. 1996.

3) Mitcheltree, R. A, Kellas, S., Dorsey, J. T., Desai, P. N., and Martin, C. J., "Passive Earth-Entry Capsules for Mars Sample Return," AIAA Paper 98-2851, June 1998. 
4) Guernsey, C.S., Thunnissen, D.P., Adler, M., French, J., "Evaluation of Some Candidate Propulsion Technologies for Mars Ascent," AIAA 98-0651, Jan. 1998.

5) “Mars Ascent Avionics Final Report FY97," JPL Interoffice Memo 311.1/97-28, Nov. 1997.

6) Bonner, E., Clever, W., and Dunn, K., "Aerodynamic Preliminary Analysis System II. Part I - Theory," NASA CR 165627, 1989.

7) Divan, P., and Sova, G., "Aerodynamic Preliminary Analysis System II. Part II - User's manual.," NASA CR 165628, 1989.

8) Treon, S. L., "Static Aerodynamic Characteristics of Short Blunt Cones With Various Base Cone Angles at mach Numbers From 0.6 to 5.5 and Angles of Attack to 180 degrees," NASA TN D-1327, May 1962.

9) Wehrend, W. R., "Wind Tunnel Investigation of the Static and Dynamic Stability Characteristics of a 10 degree Semivertex Angle Blunted Cone," NASA TN D-1202, Jan. 1962.

10) Intrieri, P. F., "Free-Flight Measurements of the Static and Dynamic Stability and Drag of a 10 degree Blunted Cone at Mach Number 3.5 and 8.5," NASA TN D-1299, Jan. 1962.

11) Anonymous, "Suitability of the Discoverer and Nerv Entry Vehicle for Mars Atmospheric Entry," General Electric Corporation, Missile and Space Vehicle Department, NASA Contract No. NAS7-100, General Electric Report No.62-322, April 1962.

12) Gnoffo, P.A., Gupta, R. N., and Shinn, J. L., "Conservation Equations and Physical Models for Hypersonic Air Flows in Thermal and Chemical Nonequilibrium," NASA TP-2867, Feb. 1989.
13) Cheatwood, F. McNeil and Gnoffo, Peter A., "User's Manual for the Langley Aerothermodynamic Upwind Relaxation Algorithm (LAURA)," NASA TM 4674, April 1996.

14) Riley, C.J. and Cheatwood, F.M., "DistributedMemory Computing with the Langley Aerothermodynamic Upwind Relaxation Algorithm (LAURA)," Proceedings of the 4th NASA National Symposium on Large-Scale Analysis and Design on High-Performance Computers and Workstations, Williamsburg, VA, October $15-17,1997$.

15) Gnoffo, P. A., Weilmuenster, K. J., Braun, R. D., and Cruz, C. I., "Influence of Sonic-Line Location on Mars Pathfinder Probe Aerothermodynamics," Journal of Spacecraft and Rockets, Vol. 33, No. 2, March-April 1996.

16) Mitcheltree, R.A., Moss, J.N., Cheatwood, F.M., Greene, F.A., and Braun, R.D., "Aerodynamics of the Mars Microprobe Entry Vehicles," AIAA Paper 97-3658, August 11-13, 1997.

17) Brauer, G. L., Cornick, D. E., and Stevenson, R., "Capabilities and Applications of the Program to Optimize Simulated Trajectories (POST)," NASA CR-2770, Feb. 1977.

18) Hargraves, C. R., and Paris, S. W., "Direct Trajectory Optimization Using Nonlinear Programming and Collocation," Journal of Guidance, Control, and Dynamics, Vol. 10, No. 4, 1987, pp. 338-342.

19) Justus, C. G., and Chimonas, "The Mars Global Reference Atmospheric Model (Mars-GRAM)," TR, Georgia Tech Project G-35-685, prepared for NASA Marshall SpaceFlight Center under Grant NAG8-078, July 1989. 